\title{
Content-based Image Retrieval using Texture and Color Features
}

\author{
Abbas Hanon AL-Asadi \\ Ali Basim AL-Khafaji \\ Computer Science Department - Science College - Basrah University - Basrah, Iraq \\ Abbashh2002@yahoo.com \\ alibasim8576@yahoo.com
}

\begin{abstract}
The emergence of multimedia technology and the rapidly expanding image collections on the Internet have attracted significant research efforts in providing tools for effective retrieval and management of visual data. Content-based image retrieval has been an active area of research over last decade. In this paper, a content-based image retrieval system is presented. It supports querying by example to retrieve images from the images database according to their texture and color features. For feature extractions, Gray-Level Co-occurrence Matrix (GLCM) and color histogram of HSV have been used. The results appeared quite satisfactory.
\end{abstract}

Keywords: Content based image retrieval, visual information, feature extraction.

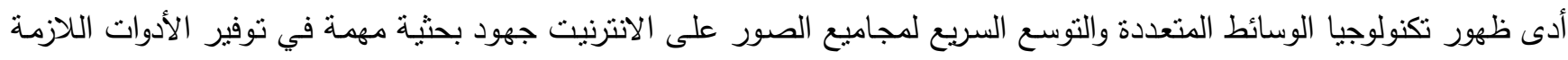

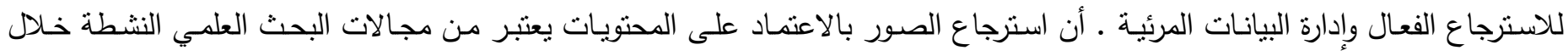

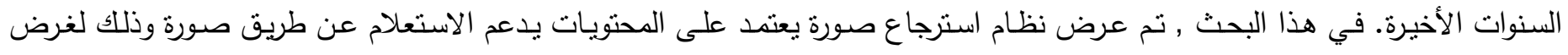

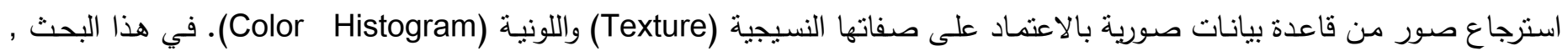

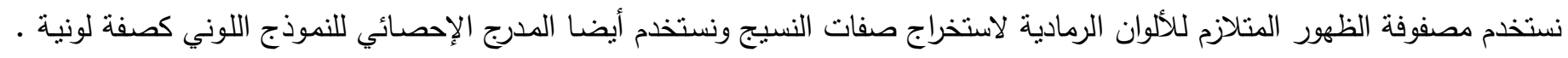
النتائج كانت مرضية جدا.

\section{Introduction}

In the early 1990s, the difficulties of using textbase image retrieval systems motivates the research into what is referred as content based image retrieval (CBIR) where retrieval is based on the automating matching of feature of query image with that of image database through home image- image similarity evaluation. Therefore images will be indexed according to their own visual content such as color, texture, shape or any other feature or a combination of set of visual features [1-3].

The earliest mention of content-based image retrieval (CBIR) appeared in the literature written by Kato in 1992, when demonstrated his experiments in automatically retrieving similar 
images for a query image from a large database using some specific features. Since then, the term CBIR has been widely used to describe the process of retrieving desired images in an image database by employing image features [4].

The application of CBIR systems are distributed in many areas. The end users of such systems range from simple users searching a particular image on the web to various types of professional bodies from the government and private organizations, for example, the police force for picture recognition, journalists requesting pictures that match some query(ies) event(s), or engineers investigating a possible anomaly in system design try to find the right mapping of initial query images. [5-7]

The visual information (contents) are the chosen features like color (distribution of color intensity across the image), texture (presence of visual patterns that have properties of homogeneity and do not result from the presence of single color or intensity), shape (boundaries, or the interiors of objects depicted in the image), or any other visual feature or a combination of a set of elementary visual features.

There are three fundamental bases for content based image retrieval, i.e. visual feature extraction, multidimensional indexing, and retrieval system design [6].

1. Feature extraction and indexing of image database according to the chosen visual features, which from the perceptual feature space, for example color, shape, texture or any combination of above.

2. Feature extraction of query image.

3. Matching the query image to the most similar images in the database according to some image-image similarity measure. This forms the search part of CBIR systems.

In this paper, our target is to build a CBIR system focusing on the local and global features of texture and color information.

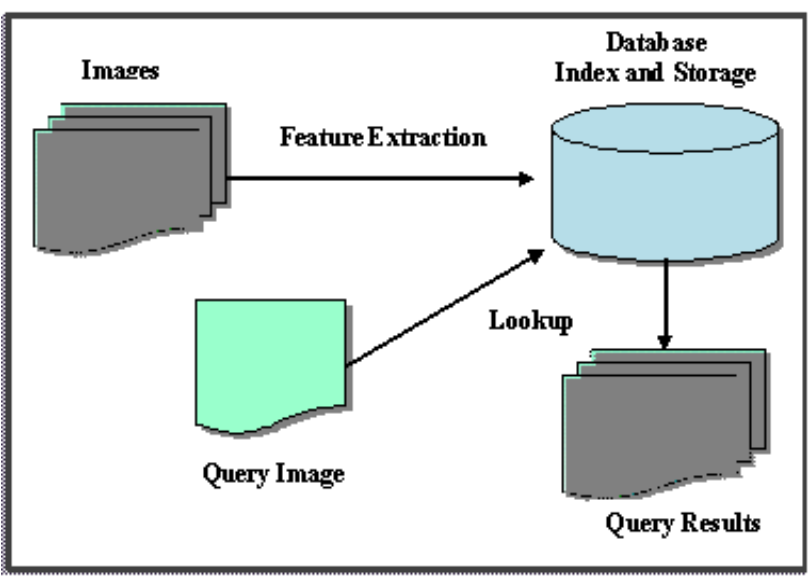

Figure (1): The essential bases of CBIR

\section{Feature Extraction}

Feature extraction is the basis of any contentbased image retrieval system. Representation of images needs to consider which features are most useful for representing the contents of the considered images, and which approaches can effectively code the attributes of the images. The sections 2.1 and 2.2 introduce two kinds of features (texture and color) which are most often used to extract the features of an image [8-10].

\subsection{Texture Feature}

Texture Feature is innate property of all surfaces that describes visual patterns, and that contains important information about the structural arrangement of the surface and its relationships to the surrounding environment (see Figure 2). The texture of an image region is determined by the way the gray levels are distributed over the pixels in the region. Image texture can be qualitatively evaluated as having one or more of the properties of randomness, lineation, or being fineness, coarseness, smoothness, granulation, mottled, irregular, or hummocky [9]. 

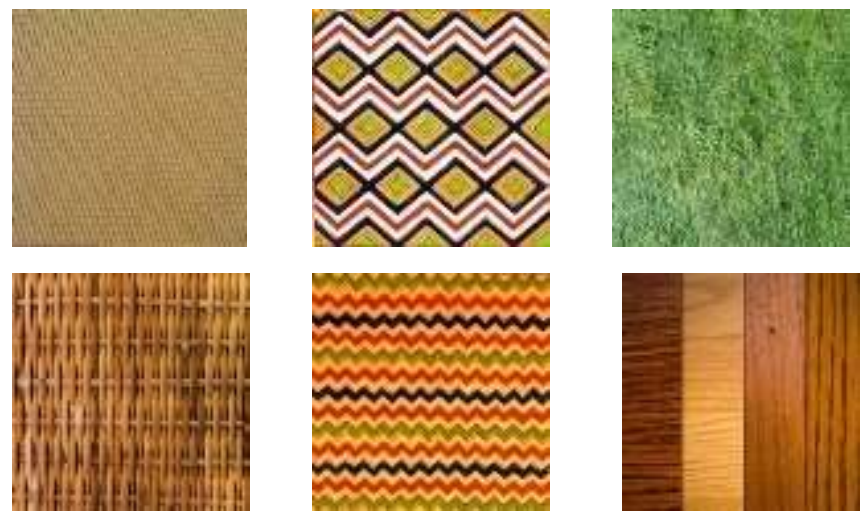

Figure (2) : Sample of Texture Feature.

Texture understanding methods are divided into three major groups [10, 11], Spatial methods, Structural methods, and Statistical methods. In this paper, worked on the statistical method.

In statistical method a texture is modeled as a random field, and a statistical probability density function model is fitted to the spatial distribution of intensities in the texture. Texture features are computed from the statistical distribution of observed combinations of intensities at specified positions relative to each other in the image.

Gray Level Co-occurrence Matrix (GLCM) is one of the most well-known statistical tools for extracting second- order texture information from images. It is a representation of the spatial distribution and the interdependence of the gray levels within a local area. The GLCM $(d, \theta)$ of an $\mathrm{NxN}$ image, containing pixels with gray levels $\{0$, $1, \ldots, \mathrm{G}-1\}$, is a two-dimensional matrix $\mathrm{P}(\mathrm{i}, \mathrm{j})$. Each element of the matrix represents the probability of joint occurrence of intensity levels $i$, $\mathrm{j}$ at a certain distance, say $\mathrm{d}$, and an angle $\theta$.

Depending on various values of $\mathrm{d}$ and $\theta$ (such as $0^{\circ}, 45^{\circ}, 90^{\circ}$ and $135^{\circ}$ ), there may be multiple numbers of co-occurrence matrices. These matrices are square, symmetric whose dimensions equal to the co-occurrence matrix number of grey levels in the picture. To generate results faster, the number of grey levels can be reduced by scaling the brightness dynamic range of the whole image, and this will reduce the dimensions of it. Symmetric means that $\mathrm{P}_{\mathrm{d}, \theta}(\mathrm{i}, \mathrm{j})=\mathrm{P}_{\mathrm{d}, \theta}(\mathrm{j}, \mathrm{i})[11]$.

Figure (3a), shows a $4 \times 4$ image with four gray levels, ranging from 0 to 3 . Figure (3b) shows the general form of any GLCM where \# denotes the number of elements. Figure (4) shows the GLCMs calculations for the four directions.

$$
\begin{array}{llll}
0 & 1 & 2 & 3
\end{array}
$$

\begin{tabular}{|c|c|c|c|c|}
\hline 0 & 0 & 1 & 1 & 1 \\
\hline 1 & 0 & 0 & 1 & 1 \\
\hline 2 & 0 & 2 & 2 & 2 \\
\hline 3 & 2 & 3 & 3 & 3 \\
\hline
\end{tabular}

a

$\begin{array}{llll}\#(0,0) & \#(0,1) & \#(0,2) & \#(0,3) \\ \#(1,0) & \#(1,1) & \#(1,2) & \#(1,3) \\ \#(2,0) & \#(2,1) & \#(2,2) & \#(2,3) \\ \#(3,0) & \#(3,1) & \#(3,2) & \#(3,3)\end{array}$

b

\section{Figure (3) :(a) 4×4 image (b) General form of GLCM}

$$
\begin{array}{cc}
P\left(0^{\circ}\right)=\left(\begin{array}{llll}
4 & 2 & 1 & 0 \\
2 & 4 & 0 & 0 \\
1 & 0 & 6 & 1 \\
0 & 0 & 1 & 2
\end{array}\right) & P\left(90^{\circ}\right)=\left(\begin{array}{llll}
6 & 0 & 2 & 0 \\
0 & 4 & 2 & 0 \\
2 & 2 & 2 & 2 \\
0 & 0 & 2 & 0
\end{array}\right) \\
P\left(135^{\circ}\right)=\left(\begin{array}{llll}
2 & 1 & 3 & 0 \\
1 & 2 & 1 & 0 \\
3 & 1 & 0 & 2 \\
0 & 0 & 2 & 0
\end{array}\right) & P\left(45^{\circ}\right)=\left(\begin{array}{llll}
4 & 1 & 0 & 0 \\
1 & 2 & 2 & 0 \\
0 & 2 & 4 & 1 \\
0 & 0 & 1 & 0
\end{array}\right)
\end{array}
$$

Figure (4) A $4 \times 4$ image with its GLCMs for $0^{\circ}, 90^{\circ}, 135^{\circ}$ and $45^{\circ}$

In order to estimate the similarity between different gray level co-occurrence matrices, many statistical features extracted from them were proposed [12]. To reduce the computational complexity, we only select five of these features as retrieval feature: Contrast, Entropy, Energy, Correlation, and homogeneity.

Contrast measures the amount of local variations in an image [6].

$$
\text { Contrast }=\sum_{i} \sum_{j}(i-j)^{2} P(i, j) \ldots \ldots .
$$

Entropy measures the disorder of an image and it achieves its largest value when all elements in $\mathrm{P}$ matrix are equal. 


$$
\text { Entropy }=\sum_{i} \sum_{j} \boldsymbol{P}(\boldsymbol{i}, \boldsymbol{j}) \log \boldsymbol{P}(\boldsymbol{i}, \boldsymbol{j}) \ldots \ldots
$$

Energy is a measure of texture uniformity of an image, the more homogeneous the image, the larger the value.

$$
\text { Energy }=\sum_{i} \sum_{j} P(i, j)^{2} \ldots \ldots
$$

The correlation feature is a measure of gray tone linear dependencies in the image.

$$
\text { Correlation }=\sum_{\boldsymbol{i}, \boldsymbol{j}} \frac{\left(\boldsymbol{i}-\mu_{\boldsymbol{i}}\right)\left(\boldsymbol{j}-\mu_{\boldsymbol{j}}\right) \boldsymbol{P}(\boldsymbol{i}, \boldsymbol{j})}{\sigma_{\boldsymbol{i}} \sigma_{\boldsymbol{j}}} \ldots \ldots
$$

Where $\mu_{\mathrm{i}}, \mu_{\mathrm{y}}, \boldsymbol{\sigma}_{\mathbf{i}}$ and $\boldsymbol{\sigma}_{\mathbf{j}}$ are the means and standard deviations.

Homogeneity is a measure of the amount of local uniformity present in the image.

$$
\text { Homogeneity }=\sum_{i j} \frac{P(i, j)}{i+|i-j|} \ldots \ldots
$$

\subsection{Color Feature}

The human eye is sensitive to colors, and color features are one of the most important elements enabling humans to recognize images. Color features are, therefore, fundamental characteristics of the content of images.

Color histograms are generally used to represent the color features of images. Before using color histograms, however, a color space model and a distance metric must be chosen and quantify [13].

Choices of color space may have significant influences on the result of image retrieval. There are many kinds of color space, including $\mathrm{YCbCr}$, YUV, HSx, CIE L*a*b*, and CIE L* $\mathrm{u}^{*} \mathrm{v}^{*}$ [14]. In spite of RGB, YCbCr, and YUV color spaces are commonly used in raw data and coding standards, they are not close to human perceptions.

HSV (Hue, Saturation and Value) and HSI (Hue, Saturation and Intensity) systems are closely related to each other and to the artist's concept of color, shade, and tone. In these systems, Hue can be described as a color property of light. It may also be conceived as a property of the surface reflecting or transmitting the light. Saturation is the measure of colorfulness or whiteness in the perceived color [15-17].
Color histogram is the most common form of the histogram is obtained by splitting the range of the data into equally sized bins. The color histogram is easy to compute and effective in characterizing both the global and local distribution of colors in an image.

Given a color image $\mathrm{I}(\mathrm{x}, \mathrm{y})$ of size $\mathrm{X} \times \mathrm{Y}$ pixels characterized by the color $\mathrm{I}$ at location $(x, y)$, i.e. $\mathrm{i}=\mathrm{I}(\mathrm{x}, \mathrm{y})$. A single bin contains the number of pixels having the color $i$. It is defined by the following equation [12]:

$$
h(i)=\frac{1}{\mathrm{xy}} \sum_{x=0}^{x-1} \sum_{y=0}^{y-1} \lambda(I(x, y), i)
$$

where

$$
\lambda(w, p)= \begin{cases}1 & \text { if } \mathrm{w}=\mathrm{p} \\ 0 & \text { otherwise }\end{cases}
$$

From the equation above, $\lambda$ is the unitary impulse function. The $h(i)$ values are normalized in order to sum to one. A histogram can be determined for each color component, resulting in three different color histograms for every single image. Another possible method is to have a single color histogram for all of the color channels. In the latter approach, the color histogram is simply a compact combination of three histograms [18].

\section{Algorithm (1) Retrieval the Most Similar}

Images For Texture and Color Feature:

Input: 100 images

Output: Texture and Color Feature Matrix

Begin

Initialize Matrices $\mathrm{G} 0=\mathrm{G} 1=\mathrm{G} 2=\mathrm{G} 4=$ zeros $(\mathrm{n}, \mathrm{m})$

For $\mathrm{i} \leftarrow 1$ To $\mathrm{m}$

For $\mathrm{j} \leftarrow 1$ To $\mathrm{m}-1$

Compute G0;

Compute G1;

Compute G2;

Compute G3;

End 


\section{End}

Compute the five features for each Matrix stats 0 = graycoprops (G0);

stats 1 = graycoprops $(\mathrm{G} 1)$;

stats2 = graycoprops (G2);

stats3 = graycoprops (G3);

feature $1(1,:)=$ [stats 0 . Contrast

stats 0 .Correlation stats 0 .Energy

stats 0 .Homogeneity entropy(G0)

stats1.Contrast stats1.Correlation

stats1.Energy stats1.Homogeneity en-

tropy(G1) stats2.Contrast

stats2.Correlation stats2.Energy

stats2.Homogeneity entropy(G2)

stats3.Contrast stats3.Correlation

stats 3 .Energy stats 3 .Homogeneity en-

tropy(G3)];

Compute the color features

$\mathrm{x}=\mathrm{x} 1$;

$\mathrm{R}=\mathrm{x}(\because,: 1)$;

$\mathrm{G}=\mathrm{x}(\because,: 2)$;

$\mathrm{B}=\mathrm{x}(\because,: 3)$;

$[\mathrm{m} \mathrm{n} \mathrm{c}]=\operatorname{size}(\mathrm{x})$;

For $\mathrm{i}=1: \mathrm{m}$

For $\mathrm{j}=1: \mathrm{n}$

$R 1=R(i, j) ; G 1=G(i, j) ; B 1=B(i, j)$;

$\mathrm{x} 1=0.5 *(\mathrm{R} 1-\mathrm{G} 1)+(\mathrm{R} 1-\mathrm{B} 1)$;

$\mathrm{zz}=\left((\mathrm{R} 1-\mathrm{G} 1)^{\wedge} 2\right)+\left((\mathrm{R} 1-\mathrm{B} 1)^{*}(\mathrm{G} 1-\mathrm{B} 1)\right) ;$

x2 =sqrt (double(zz));

the $=\cos ($ double $(x 1 / x 2))$;

IF $\mathrm{B} 1>\mathrm{G} 1$

$H(i, j)=2 * 3.14-$ th;

\section{Else}

\section{End}

$\mathrm{H}(\mathrm{i}, \mathrm{j})=\mathrm{th}$;

$$
\begin{aligned}
& \mathrm{z} 1=[\mathrm{R} 1, \mathrm{G} 1, \mathrm{~B} 1] \\
& \mathrm{S}(\mathrm{i}, \mathrm{j})=1-\left(\left(3^{*} \min (\mathrm{z} 1)\right) /(\mathrm{R} 1+\mathrm{G} 1+\mathrm{B} 1)\right) \\
& \mathrm{V}(\mathrm{i}, \mathrm{j})=(\mathrm{R} 1+\mathrm{G} 1+\mathrm{B} 1) / 3
\end{aligned}
$$

\section{End}

End

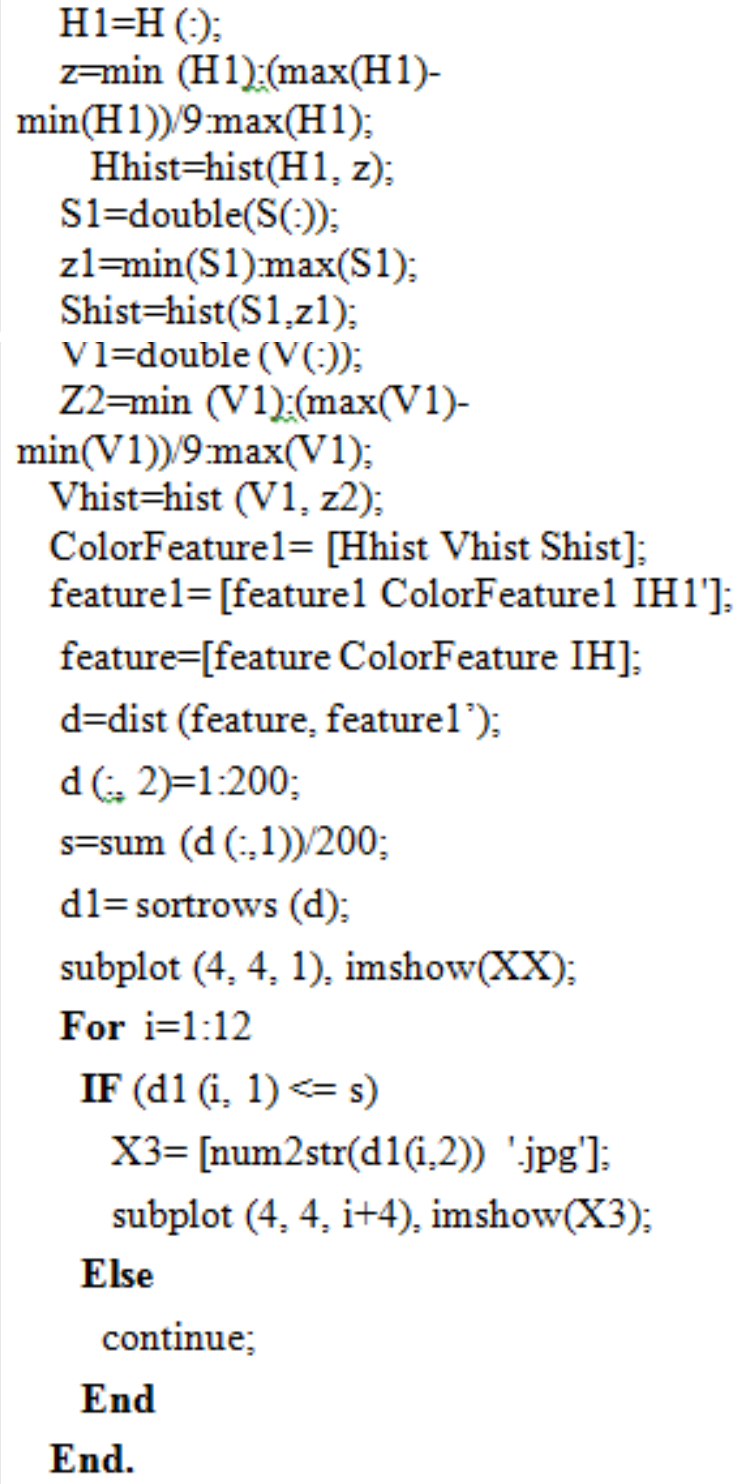

End. 


\section{Results and Discussion}

In our work, Content Based Image Retrieval (CBIR) using Texture and Color experiment is performed on a color image database. This database contains two classes of images (tiger and rose). Each class contains 20 images. Algorithm (1) displays the main steps of proposed CBIR system.

Figures (5, 6, and 7) show the results of our proposed method. Tables (1 and 2) appear some of our calculations of the feature of texture and color respectively.

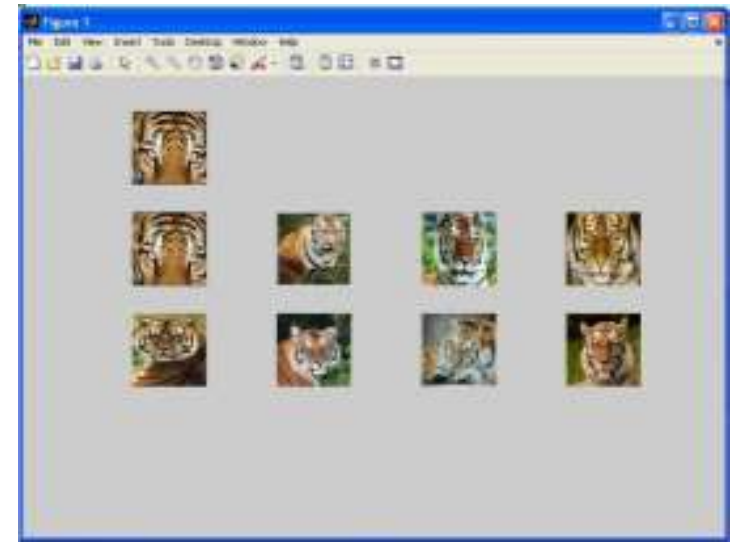

Figure (5): shows the retrieval results obtained using texture features for the query image 'tiger'

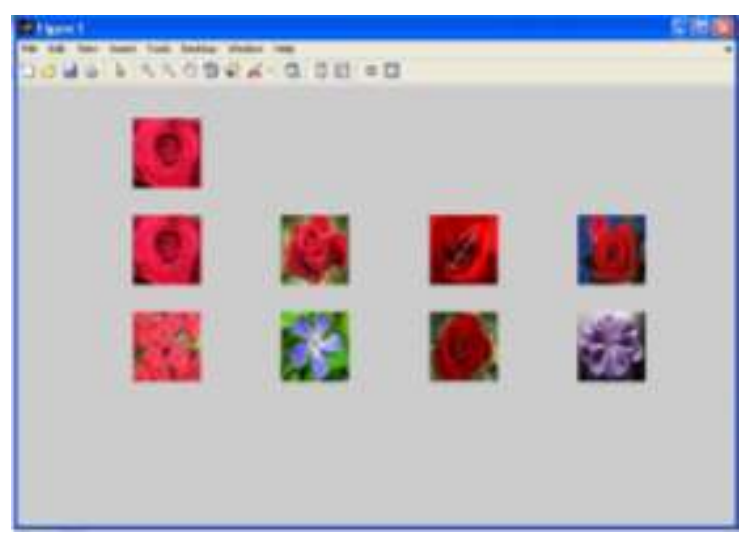

Figure (6): shows the retrieval results obtained using color feature for the query image 'flower'

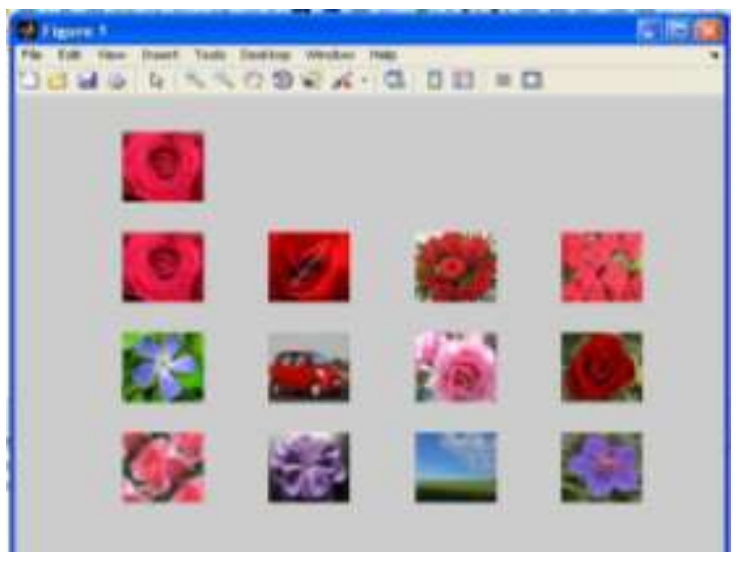

Figure (7): shows the retrieval results obtained using Texture and color feature for the query image 'flower'

Table (1) Performance of Different Texture Features

\begin{tabular}{|c|c|c|c|c|c|}
\hline Contrast & Correlation & Energy & Entropy & Homogeneity \\
\hline 1 & 0.543 & 0.373 & 0.001 & 0.953 & 96.020 \\
\hline 2 & 0.483 & 0.454 & 0.001 & 0.834 & 177.205 \\
\hline 3 & 0.467 & 0.508 & 0.002 & 0.973 & 54.542 \\
\hline 4 & 0.609 & 0.238 & 0.000 & 0.959 & 214.452 \\
\hline 5 & 0.444 & 0.425 & 0.001 & 0.988 & 144.5007 \\
\hline 6 & 0.589 & 0.221 & 0.001 & 0.906 & 484.244 \\
\hline 7 & 0.609 & 0.237 & 0.003 & 0.935 & 245.260 \\
\hline 8 & 0.872 & 0.269 & 0.005 & 0.910 & 134.593 \\
\hline 9 & 0.661 & 0.158 & 0.002 & 0.752 & 430.070 \\
\hline 10 & 0.861 & 0.114 & 8.904 & 0.819 & 1584.294 \\
\hline
\end{tabular}

Table (2) Performance of Color Features

\begin{tabular}{|c|c|c|c|c|c|c|}
\hline \multirow{2}{*}{ Type } & \multicolumn{2}{|c|}{ Hue(H) } & \multicolumn{2}{c|}{ Saturation(S) } & \multicolumn{2}{c|}{ Value(V) } \\
\hline 1 & 9273 & 732 & 1663 & 14721 & 3658 & 700 \\
\hline 2 & 2815 & 865 & 7057 & 9327 & 54 & 1 \\
\hline 3 & 13104 & 24 & 2819 & 13565 & 8 & 1 \\
\hline 4 & 5973 & 8603 & 8620 & 22236 & 2627 & 1164 \\
\hline 5 & 13923 & 883 & 10923 & 89361 & 474 & 20 \\
\hline 6 & 37 & 99 & 28953 & 93459 & 1615 & 19 \\
\hline 7 & 29736 & 157 & 30390 & 92022 & 1742 & 3080 \\
\hline 8 & 90224 & 2095 & 27285 & 95127 & 1237 & 19 \\
\hline 9 & 122351 & 4 & 29211 & 93201 & 1382 & 19 \\
\hline 10 & 119261 & 2980 & 28310 & 94102 & 1314 & 63 \\
\hline
\end{tabular}




\section{Conclusion}

The area of content-based image retrieval is a research area that requires knowledge of both computer vision and of database systems. The application of information theory to image interpretation and CBIR poses many questions for further exploration.

The field appears to be generating interesting and valid results, even though it has so far led to few commercial applications. Agencies concerned with technology transfer or dissemination of best practice in fields which could potentially benefit from CBIR (including management of image collections and drawing archives, electronic publishing and multimedia content creation) should consider sponsoring programmers to raise awareness of CBIR technology among leading practitioners in these fields.

\section{References}

1. Smeulders, A. W., Worring M., Santini S., Gupta A. and Jain R. Fellow, ContentBased Image Retrieval at the End of the Early Years, Transactions on Pattern Analysis and Machine Intelligence IEEE, 22(12):1-32, 2000.

2. Konak E.S., A Content-Based Image Retrieval System for Texture and Color Queries, M.Sc. thesis in Computer Engineering, Institute of Engineering and Science, Bilkent university, Turkey, August, 2002.

3. Gang Zhang and Zong-Min Ma. Texture Feature Extraction and Description Using Gabor Wavelet in Content-Based MedicaII mage Retrieval, Proceedings of the International Conference on Wavelet Analysis and Pattern Recognition, Beijing, China, 2-4 Nov. pp 169-173, 2007.

4. Egon L. van den Broek, Human-Centered Content-Based Image Retrieval,
Netherlands Organization for Scientific Research (NWO), 2005.

5. W. M. Smeulders, M. Worring, S. Santini, A. Gupta, and R. Jain, Content-Based Image Retrieval at the End of the Years. IEEE Transactions on Pattern Analysis and Machine Intelligence, 22(12):1349-1380, December 2000.

6. Jiawei Han and Micheline Kamber, Data Mining Concepts \& Techniques, Morgan Kaufmann Publishers, 2006.

7. A. Grace Selvarani and S. Annadurai, Content Based Image Retrieval For Medical Images Using Generic Fourier Descriptor , Journal of Computational Intelligence in Bioinformatics.1(1) : 65-72, 2008

8. Chang C. C., $\mathrm{Wu} \mathrm{W.} \mathrm{C.} \mathrm{and} \mathrm{Hu}$ Y. C., Content-Based Color Image Retrieval System Using Color Difference Features, Second International Conference on Future Generation Communication and Networking Symposia IEEE, 10 ():181$184,2008$.

9. Chun Y. D., Kim N. C. and Jang I. H., Content-Based Image Retrieval Using Multiresolution Color and Texture Features, transactions on multimedia IEEE, 10(6):1073-1084, 2008.

10. Acharya T. and Ray A. K., Image Processing: Principles and Application, John Wiley \& Sons, Inc, Hoboken, New Jersey, 2005.

11. Haralick R. M., Statistical and Structural Approaches to Texture, Proceeding of the IEEE, 67 (5):786-808, 1979.

12. Partio, M., Cramariuc, B., Gabbouj,M., Texture retrieval using ordinal cooccurrence features, Proceedings of the 6th Nordic Signal Processing SymposiumNORSIG 2004, pp.308 - 311 
13. Wang S., A Robust CBIR Approach Using Local Color Histograms, M.Sc. Thesis, Department of Computing Science, University of Alberta, Canada, 2001.

14. Rastislav Lukac and Konstantinos N., Color Image Processing Methods and Applications, CRC Press, Taylor \& Francis Group, 2007.

15. Ratt W. K., Digital Image Processing, $3^{\text {rd }}$ Edition, John Wiley \& Sons, Inc, New York, 2001.

16. Gonzalez R. C., Woods R. E. and Eddins S. L., Digital Image Processing Using Matlab, John Wiley \& Sons, Inc, 2003.

17. Russ J. C., The Image Processing Handbook, $5^{\text {th }}$ Edition, Taylor \& Francis Group, LLC, 2007.

18. Boujemaa N., Boughorbel S., and Vertan C., Soft Color Signatures for Image Retrieval by Content, INRIA Rocquencourt, IMEDIA Project, Cedex, France, 2001. 